\title{
Miocardiopatía séptica: abordaje desde la sala de urgencias
}

\author{
Bárbara Mónica Rodríguez-Camacho ${ }^{1}$, María Elena Bello-Méndez¹, Marco Antonio Sosa-Ramírez e \\ Iván Mauricio Lima-Lucero ${ }^{2 *}$
}

${ }^{1}$ Servicio de Urgencias, Hospital General Balbuena; ${ }^{2}$ Unidad de Terapia Intensiva Cardiovascular, Instituto Nacional de Cardiología Dr. Ignacio Chávez. Ciudad de México, México

\section{Resumen}

La miocardiopatía séptica es una patología mal definida, de características clínicas variables, con incidencia incierta y de difícil diagnóstico; este manuscrito expone una revisión de los conceptos básicos, recordando los fenómenos de la respuesta inflamatoria desproporcionada a una infección y sepsis con impacto a nivel cardiaco. Se analiza el monitoreo y abordaje de diagnóstico clínico, bioquímico y ecográfico que guiará al especialista en medicina de urgencias hacia el tratamiento ideal de esta compleja disfunción orgánica. Para la realización de la presente revisión se realizó una búsqueda exhaustiva en PubMed y ScienceDirect de la evidencia más recientemente publicada.

Palabras clave: Sepsis. Insuficiencia cardiaca. Medicina de Emergencia.

\section{Septic myocardiopathy: approach from the Emergency Room}

\begin{abstract}
Septic cardiomyopathy is a poorly defined pathology with variable clinical characteristics, with uncertain incidence and difficult diagnosis; this manuscript exposes a review of the basic concepts, recalling the phenomena of the inflammatory response disproportionate to infection and sepsis with impact at cardiac level. The monitoring and clinical diagnosis, biochemical and ultrasound approach that will guide the specialist in Emergency Medicine towards the ideal treatment of this complex organic dysfunction are analyzed.
\end{abstract}

Key words: Sepsis. Heart failure. Emergency Medicine.

\section{Introducción}

La presencia de repercusión hemodinámica es una de las primeras manifestaciones de alteración sistémica en el paciente séptico. Además del componente vasodilatador y cambios de precarga y poscarga, el compromiso de la función cardiaca por el estrés generado debido al grado de inflamación lleva a perpetuar el estado de hipoperfusión sistémica.

Aunque identificado desde hace mucho tiempo, no fue sino hasta la caracterización de la fisiopatología molecular de la sepsis, la interrelación de los antígenos

\section{Correspondencia:}

*Iván Mauricio Lima-Lucero

E-mail: ivanlima.urgencias@gmail.com
Fecha de recepción: 02-02-2019

Fecha de aceptación: 25-09-2019 DOI: 10.24875/REIE. 19000012
Disponible en internet: 06-11-2019 Rev Educ Investig Emer. 2019;1(3):98-105 www.medicinadeemergencias.com

2604-6520 @ 2019 Sociedad Mexicana de Medicina de Emergencias, AC. Publicado por Permanyer México SA de CV. Este es un artículo open access bajo la licencia CC BY-NC-ND (http://creativecommons.org/licenses/by-nc-nd/4.0/). 
con los receptores tipo Toll (TLR) y el desencadenamiento de la cascada inflamatoria que se han podido definir y generar estrategias de monitorización y tratamiento de esta entidad.

El abordaje en el área de urgencias del paciente con miocardiopatía séptica es sumamente importante, ya que su reconocimiento temprano permitirá abordar de manera sistemática y adecuada al paciente, monitorizarlo mediante diferentes técnicas, y hacer uso de la ecocardiografía a la cabecera del paciente, para dar el seguimiento en las primeras horas.

\section{Definición}

En los últimos 50 años se han evaluado las alteraciones cardiovasculares en pacientes con sepsis'; el término de depresión miocárdica secundaria a sepsis se introdujo en 1984 por Parker, quien observó en una serie de evaluaciones hemodinámicas de 20 pacientes con shock séptico, que los pacientes con índice cardiaco (IC) normal presentaban una reducción de la fracción de eyección del ventrículo izquierdo $(\mathrm{FEVI})^{2}$. En los últimos 20 años se ha definido la miocardiopatía séptica como una alteración reversible de la función cardiovascular con disminución de la fracción de eyección (FE) de ambos ventrículos, y que se puede acompañar de dilatación ventricular con pobre respuesta a la reanimación con líquidos y vasopresores ${ }^{3}$. En la actualidad se cuenta con diversas definiciones basadas en sus manifestaciones, como: insuficiencia ventricular izquierda y/o derecha, sistólica o diastólica, gasto cardiaco (GC) inadecuado para el suministro de oxígeno y lesión celular miocárdica primaria ${ }^{4}$. Para diferenciar unas de otras se pueden dividir en dos grandes grupos: ecocardiográficas y no ecocardiográficas 4 . Dentro de las ecocardiográficas podemos mencionar al ventrículo izquierdo (VI) en su función sistólica, siendo la FE la de uso habitual, con valores de anormalidad de $<52 \%$ en hombres $0<54 \%$ en mujeres ${ }^{5}$, este parámetro es usado frecuentemente, fácil de adquirir, puede variar de un latido a otro, y es muy dependiente de las condiciones de precarga del Vl; además no logra identificar con precisión a todos los pacientes con miocardiopatía séptica ${ }^{6,7}$. La disfunción del ventrículo derecho (VD) también ocurre en la sepsis, generalmente en conjunto con la disfunción del $\mathrm{Vl}^{8}$.

La disfunción diastólica también se ha observado en muchos pacientes con sepsis ${ }^{4} \mathrm{y}$ con frecuencia cumple con los criterios de referencia para la disfunción diastólica del VI emitida por la Sociedad Americana de Ecocardiografía?
La definición no ecocardiográfica de la disfunción miocárdica por sepsis se encuentra fundamentada en dos pilares básicamente: 1) biomarcadores químicos (troponina T y BNP), y 2) marcadores hemodinámicos (taquicardia, entrega inadecuada de oxígeno en donde se toma en cuenta el lactato y más recientemente el índice de shock y el índice de shock modificado) ${ }^{9}$.

\section{Marcadores hemodinámicos}

El shock séptico se considera un estado hiperdinámico en el cual se presenta taquicardia y aumento del GC, junto con una baja resistencia vascular sistémica. La taquicardia en sepsis es una respuesta compensatoria secundaria a un volumen de llenado cardiaco bajo, estimulación adrenérgica y la presencia de fiebre, lo cual incrementa la demanda de oxígeno y se acorta la diástole, lo que da como resultado un bajo llenado del ventrículo, a medida que el GC disminuye, se van activando los mecanismos de compensación en el sistema cardiovascular, motivo por el cual y con base en lo previamente comentado no solo se cuenta con biomarcadores como el Péptido Natriurético Cerebral (BNP) o las troponinas, sino que también se han propuesto el índice de shock y el índice de shock modificado, que permiten el reconocimiento precoz de esta disfunción hemodinámica ${ }^{6}$.

El índice de shock se calcula a partir de la división de la frecuencia cardiaca entre la presión sistólica. Recientemente se han investigado y reconocido las limitaciones de este índice de shock, motivo por el cual se ha propuesto el índice de shock modificado, en el cual se incorpora la presión arterial media (PAM); se calcula dividiendo la frecuencia cardiaca entre la PAM, representando así la función diastólica además de la sistólica. Un valor de un índice de shock modificado mayor de 1.3 se ha asociado a un aumento de la mortalidad, sin embargo no se relaciona con el incremento de los biomarcadores ni con la gravedad del paciente con sepsis?.

\section{Incidencia}

Por todas aquellas variables que dificultan la definición de miocardiopatía séptica, se tienen variaciones en la incidencia de esta patología, pero en los últimos 10 años se han realizado diversos estudios que nos muestran un panorama más preciso de esta entidad. En 2008 Vieillard evaluó la incidencia real de la hipocinesia ventricular izquierda global en shock séptico mediante ecocardiografía transesofágica y se encontró 
con una tasa global del $60 \%{ }^{10}$. Pulido en el 2012 determinó la frecuencia y el espectro de la disfunción miocárdica para evaluar el impacto en la mortalidad, mediante un estudio prospectivo, incluyendo $106 \mathrm{pa}-$ cientes; la frecuencia de disfunción miocárdica fue del $64 \%$, la disfunción diastólica del VI del 37\%, la disfunción sistólica del VI del 27\% y la disfunción del VD del $31 \%{ }^{11}$. En ese mismo año Giora evaluó una cohorte de 262 pacientes de la unidad de cuidados intensivos (UCl). La disminución de la velocidad en la onda e-mitral fue el predictor más fuerte de mortalidad. Los pacientes que además presentaban disfunción diastólica presentaron mayor mortalidad que aquellos sin disfunción diastólica o sistólica aislada y tuvieron niveles séricos significativamente más altos de troponina $\mathrm{T}$ de alta sensibilidad y de tipo N-terminal pro-B ${ }^{12}$.

Brown buscó determinar si la gravedad de la disfunción diastólica evaluada mediante ecocardiografía transtorácica (TTE) para predecir mortalidad a los 28 días, mediante un estudio prospectivo, observacional, con 78 pacientes que se sometieron a TTE dentro de $6 \mathrm{~h}$ de ingreso en la $\mathrm{UCl}$, después de 18 a $32 \mathrm{~h}, \mathrm{y}$ después de la resolución del shock; como resultados en el $36.5 \%$ se identificó disfunción diastólica en el ecocardiograma inicial y el $61.5 \%$ en al menos un estudio a lo largo de su hospitalización. La mortalidad fue del $16.5 \%$, pero subió al $37.5 \%$ cuando se añadía además disfunción diastólica grado I ${ }^{13}$. En 2016 Sato evaluó la epidemiología y las características clínicas de la miocardiopatía inducida por sepsis (CMS) en un estudio retrospectivo, con 210 pacientes; la definición de CMS fue $\mathrm{FE}<50 \%$ y una disminución $\geq 10 \%$ en comparación con $\mathrm{FE}$ de base que se recuperó dentro de dos semanas, con una incidencia del $13.8 \%$, la prevalencia fue mayor en hombres, con mayor lactato al ingreso e historia de insuficiencia cardiaca, a los 30 días la mortalidad fue del $24.1 \%$, con estancia promedio de 43 días $^{14}$.

\section{Fisiopatología}

La primera hipótesis sobre el mecanismo de la disfunción miocárdica en sepsis propuso a la isquemia global del miocardio como resultado de un flujo sanguíneo coronario inadecuado. Sin embargo, más tarde se descubrió que el flujo sanguíneo coronario se conservaba o aumentaba en pacientes con shock séptico y con disfunción miocárdica, refutando la hipótesis anterior. Aunque algunos estudios demostraron que hubo un deterioro en la microcirculación cardiaca durante la sepsis, debido a una mala distribución significativa del flujo sanguíneo coronario, daño endotelial, depósitos intravasculares de fibrina e infiltración de neutrófilos, lo que podría resultar en isquemia focal del miocardio y disminución de la función cardiaca, no se confirmó hipoxia miocárdica ${ }^{15}$. Los últimos estudios clínicos han demostrado que la necrosis miocárdica es infrecuente y que la depresión miocárdica es reversible, por lo que se plantea que la depresión miocárdica se debe más a alteraciones funcionales que estructurales.

La insuficiencia cardiovascular debida a sepsis implica, en general, disfunción vascular periférica y disfunción miocárdica. La disfunción vascular periférica incluye venodilatación, vasodilatación arterial, regulación alterada de la distribución del flujo sanguíneo arteriolar, heterogeneidad del flujo de la microcirculación capilar, inflamación del endotelio y la microcirculación y mayor permeabilidad de los vasos que conducen al edema tisular e hipovolemia intravascular ${ }^{15}$. En este contexto, la disfunción miocárdica se convierte en particularmente importante porque la disfunción vascular periférica genera una demanda mucho mayor en el corazón. Sin embargo, a pesar del aumento del GC y un volumen normal de eyección, la disfunción miocárdica es significativa en pacientes con shock séptico. En particular, la FE es más baja y el volumen diastólico final es más alto, lo que sugiere que la dilatación ventricular podría ser un mecanismo compensatorio para mantener el GC adecuado y proteger contra la depresión del miocardio ${ }^{16}$.

La patogenia de la depresión miocárdica en la sepsis es el resultado de una interacción compleja entre alteraciones genéticas, moleculares, metabólicas, estructurales, autonómicas y hemodinámicas ${ }^{1}$. A nivel celular diversos patrones moleculares asociados a patógenos (PAMP), como los lipopolisacáridos (LPS) y los patrones moleculares asociados a daño (DAMP) endógenos, incluidos el cuadro de grupo de alta movilidad 1 y las histonas extracelulares, interactúan con TLR en células inmunitarias y en los cardiomiocitos ${ }^{17}$.

Los cardiomiocitos son células diferenciadas dedicadas a la contracción altamente coordinada. Esta función contráctil de los cardiomiocitos se ve afectada por la forma en que reconocen y responden a las señales de peligro (principalmente los componentes de la pared celular bacteriana) con una respuesta inflamatoria y funcional compleja. A los pocos minutos de reconocer una infección bacteriana a través de una membrana celular inmune innata por los TLR, los cardiomiocitos secretan citocinas proinflamatorias y quimiocinas que 


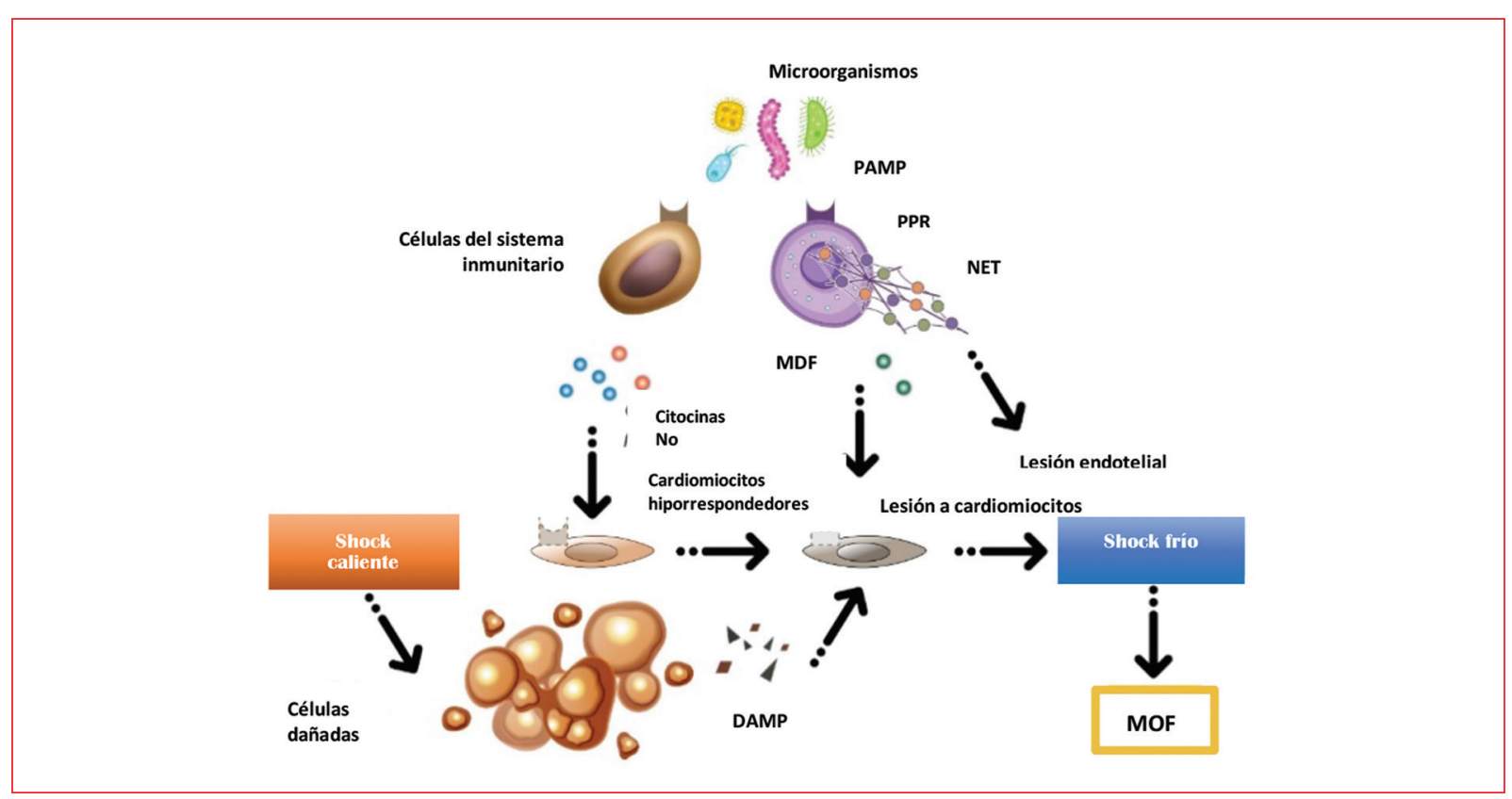

Figura 1. Depresión directa del miocardio en sepsis (adaptada de Russell, et al., 201818). DAMP: patrones moleculares asociados al daño; NET: trampas extracelulares de neutrófilos; MDF: factores de depresión del miocardio; MOF: fallo multiorgánico; PAMP: patrones moleculares asociados a patógenos;

PRR: receptores de reconocimiento de patrones.

inician una respuesta inflamatoria local y reclutan células inflamatorias ${ }^{18}$ (Fig. 1).

Anteriormente muchos autores informaron la presencia de un factor depresor del miocardio que podría inducir disfunción miocárdica en modelos experimentales de sepsis, sin embargo, los esfuerzos para identificar un solo factor depresor fallaron, y estudios posteriores analizaron una mezcla de sustancias intrínsecas. Los principales mediadores inflamatorios que podrían contribuir a la depresión miocárdica en la sepsis son: factor de necrosis tumoral alfa (TNF- $\alpha$ ), interleucina (IL) 1 e IL-6. Es probable que exista una interacción entre TNF- $\alpha /$ IL-1 y sintasa inducible de óxido nítrico (iNOS), lo que crea un efecto inotrópico negativo en el corazón. El papel de la IL-6 en la depresión miocárdica séptica se observó in vitro utilizando miocitos cardiacos ventriculares aislados de rata donde se demostró una conexión molecular entre los niveles de IL-6 y los efectos inotrópicos negativos a través de una vía de proteína cinasa ${ }^{19}$ (Tabla 1 y Fig. 1).

Por otra parte, se ha observado que la participación de los canales de calcio en la depresión miocárdica puede estar relacionada con la relación entre las concentraciones de calcio intracelular y la contracción cardiaca, por reducción de los receptores de dihidropiridina cardiacos (es decir, los canales de calcio de tipo L) durante la
Tabla 1. Factores depresores de miocardio

\begin{tabular}{|l|l|}
\hline Clasificación & Factor depresor \\
\hline Citocinas & $\begin{array}{l}\text { Interleucina-1 } \\
\text { Interleucina-6 } \\
\text { Factor de necrosis tumoral alfa }\end{array}$ \\
\hline $\begin{array}{l}\text { Componentes del } \\
\text { complemento }\end{array}$ & $\begin{array}{l}\text { Complemento activado 3 } \\
\text { Complemento de anafilatoxina (C5a) }\end{array}$ \\
\hline PAMP & LPS \\
\hline DAMP & $\begin{array}{l}\text { Cuadro de grupo de alta movilidad 1 } \\
\text { Histonas extracelulares }\end{array}$ \\
\hline $\begin{array}{l}\text { Metaloproteinasas } \\
\text { de matriz }\end{array}$ & Metaloproteinasa-9 \\
\hline
\end{tabular}

DAMP: patrones moleculares asociados al daño; PAMP: patrones moleculares asociados a patógenos; LPS: lipopolisacáridos.

endotoxemia inducida. Durante el shock séptico hay una corriente de calcio de tipo $L$ reducida y por consiguiente acortamiento de la repolarización cardiaca ${ }^{20}$.

El papel del óxido nítrico (NO) es bien reconocido en la sepsis, induce vasodilatación con cambios resultantes en la precarga, la poscarga y la perfusión cardiaca. También tiene un efecto directo sobre los cardiomiocitos, probablemente induciendo depresión del miocardio. Algunos estudios han demostrado una relación entre la 
inhibición del óxido nítrico sintasa (NOS) y la recuperación de la función miocárdica, independientemente del TNF- $\alpha$ y los niveles de IL-1, así mismo que la producción intramitocondrial de causa el agotamiento del glutatión. Es probable que el peroxinitrito producido por el metabolismo del NO afecte la función de los poros de transición de la permeabilidad mitocondrial, con la disfunción mitocondrial subsiguiente implicada en la depresión del miocardio observada durante la sepsis ${ }^{21}$.

El papel exacto de la endotelina-1 (ET-1) en la patogenia de la disfunción miocárdica séptica no está claro, se sabe que la ET-1 afecta la contractilidad miocárdica y probablemente aumenta la hipertrofia miocárdica. Durante la sepsis, los niveles de ET-1 aumentan en la sangre y en el tejido miocárdico asociado con disfunción miocárdica, posiblemente relacionada con la apoptosis de cardiomiocitos ${ }^{22}$.

Finalmente las autopsias humanas han revelado que existen varias modificaciones estructurales en el miocardio séptico. La infiltración miocárdica por las células inmunitarias, especialmente las células polimorfonucleares (PMN) y los monocitos/macrófagos, es una característica reconocida. La hinchazón marcada de células endoteliales cardiacas, la formación de trombos de fibrina en los vasos sanguíneos y la infiltración de PMN puede formar la base del edema inflamatorio del miocardio durante la sepsis. Aunque la necrosis miocárdica y la apoptosis pueden jugar un papel en la determinación de la disfunción cardiaca, la mayoría de los estudios han informado que la depresión miocárdica relacionada con sepsis fue reversible en los pacientes que sobrevivieron.

\section{Monitorización hemodinámica}

La monitorización por medio de catéter de la arteria pulmonar ayuda a detectar la depleción de aporte de oxígeno mediante la medición de la saturación mixta de oxígeno. Debido a los posibles eventos adversos derivados del uso ${ }^{23}$ del catéter de la arteria pulmonar este no se usa de manera sistemática en los pacientes críticos, a excepción de los posquirúrgicos cardiovasculares $^{24}$. El catéter de la arteria pulmonar puede ser particularmente útil en el contexto de falla ventricular derecha, hipertensión pulmonar y cirugía cardiovascular mayor ${ }^{25}$.

Actualmente no existe un escenario específico para su uso en el paciente con shock séptico, aunque algunos autores consideran la monitorización multimodal, incluyendo su uso en conjunto con ecocardiografía en el contexto de miocardiopatía séptica ${ }^{26}$.
El análisis de la onda de pulso se ha propuesto como una alternativa menos invasiva para monitorizar el GC. Sin embargo, este método es altamente dependiente de las resistencias vasculares sistémicas, las mismas que varían considerablemente durante la sepsis, en parte debido a la infusión continua con vasopresores. Es debido a esto que el uso de métodos que analicen el GC basándose en la onda de pulso no son fiables para el diagnóstico de miocardiopatía séptica ${ }^{27}$.

La realización de un electrocardiograma de 12 derivaciones añadido al resto de monitoreo siempre se debe realizar ante la sospecha de disfunción cardiaca. Los cambios electrocardiográficos en miocardiopatía séptica no son específicos y muchos de ellos pueden ser similares a los que se presentan en eventos isquémicos $^{28}$. El desarrollo de arritmias supraventriculares incluyendo fibrilación auricular son frecuentes en el estado de shock séptico.

\section{Marcadores séricos}

Adicionalmente a los marcadores convencionales de infección e inflamación, como procalcitonina, proteína $C$ reactiva y recuento de leucocitos, algunos marcadores de falla cardiaca como el péptido natriurético $\mathrm{N}$ terminal pro-tipo B (NT-pro-BNP) han sido sugeridos como adecuados para facilitar el diagnóstico de miocardiopatía séptica $^{29}$. Un BNP plasmático > $190 \mathrm{ng} / \mathrm{l}$ puede diferenciar a sobrevivientes de no sobrevivientes, con una sensibilidad del $70 \%$ y una especificidad del $67 \%$. Investigaciones recientes llevadas a cabo en 900 pacientes reveló que el NT-pro-BNP y la troponina cardiaca $T$ de alta sensibilidad se correlacionan con el desarrollo de shock séptico, independientemente de que desarrollen o no miocardiopatía séptica ${ }^{10}$.

La elevación de la troponina está relacionada con mayor requerimiento de inotrópicos, índice de trabajo ventricular más bajo, disminución de la fracción de expulsión y con una mayor mortalidad debido a que se relaciona con la necrosis de los miocitos y a disfunción del aparato contráctil. Aunque la causa de la disfunción miocárdica en la sepsis sigue sin estar clara, se cree que la troponina puede ser liberada por las células miocárdicas dañadas por el proceso infeccioso, o posiblemente por las células del miocardio debido al incremento de la demanda de oxígeno en pacientes sépticos ${ }^{4}$.

Aunque la asociación del aumento de la troponina $T$ y la disfunción miocárdica está claramente presente, aún no está claro si los enfoques del tratamiento más agresivos mejorarían la mortalidad en estos pacientes ${ }^{5}$. 
En esta investigación el NT pro-BNP fue superior a la troponina para predecir mortalidad a los 90 días en paciente con shock séptico, sin embargo ninguno de los dos tuvieron la especificidad adecuada para diagnosticar miocardiopatía séptica ${ }^{30}$.

\section{Ecocardiografía}

Estimar la función cardiaca en el paciente séptico es complicado sin tomar en consideración las variables hemodinámicas sistémicas, las cuales se pueden alterar de manera notable durante el desarrollo de una infección. Ningún tipo de monitorización aislada puede determinar al mismo tiempo la función cardiaca y variables hemodinámicas ${ }^{31}$. Además es de resaltar que al evaluar la función cardiaca debemos tener en cuenta la función sistólica y diastólica tanto del VI como del VD. Los parámetros que podemos obtener derivados de un estudio ecocardiográfico como la FEVI dependen en gran medida de la precarga y poscarga del paciente. Técnicas más avanzadas para evaluar la contractilidad ventricular como el índice de performance miocárdico o el índice de velocidad miocárdico depende mucho de una adecuada alineación del haz de ultrasonido, lo cual limita su valoración entre operadores sin contar además de las complicaciones propias del paciente en estado crítico, como posición, ventilación mecánica, presencia de electrodos y sondas. Una nueva técnica ecocardiográfica que permite determinar la función cardiaca emplea el uso de deformación en el Doppler tisular o speckle traking (ST), el cual puede ser procesado por el ecocardiografista de manera retrospectiva y no depende de ciertas angulaciones durante las mediciones ${ }^{32}$.

Comparado con la FEVI, el ST depende mucho menos de cambios en la precarga o poscarga, así como de la distensibilidad miocárdica debido a que cuantifica de manera directa la deformación miocárdica. Se han desarrollado algunos estudios en donde se utiliza esta técnica para el diagnóstico de cardiomiopatía séptica, con resultados bastante alentadores, sin embargo la evidencia aún es insuficiente para su recomendación total ${ }^{33}$.

\section{Rendimiento cardiaco relacionado con poscarga}

Con la ecocardiografía se tiene una gran herramienta para ser más certeros en el diagnóstico de miocardiopatía séptica, sin embargo también tiene la desventaja de ser un estudio no continuo. En este aspecto, el rendimiento cardiaco relacionado con poscarga (ACP) se ha propuesto como una opción para una monitorización continua de la función cardiaca. Este parámetro se expresa en porcentaje y depende del GC, la PAM y la presión venosa central (PVC), dependiendo de la siguiente ecuación ${ }^{34,35}$ :

$$
\text { ACP \% }=\frac{100 \times \text { GC }}{560.68 \times\left(\begin{array}{l}
(\mathrm{PAM}-\mathrm{PVC}) \\
\mathrm{x} 80 / \mathrm{GC}
\end{array}\right)^{-0.645}}
$$

En conjunto, resulta interesante que solo un abordaje multimodal permite un diagnóstico adecuado, clasificación y monitorización del paciente con miocardiopatía séptica. La clasificación y monitorización del funcionamiento cardiaco se verán facilitadas mediante el abordaje mediante el examen físico y monitorización hemodinámica con métodos invasivos y no invasivos incluyendo el uso de nuevas técnicas, entre ellas ACP, ST e índice de performance miocárdica.

\section{Manejo}

Además del tratamiento dirigido hacia la causa base, se generan teorías dirigidas hacia el sustento fisiopatológico del aumento del consumo de oxígeno a nivel miocárdico en correlación al IC. Varios estudios han evaluado el incremento de IC a valores supranormales (IC > $4.5 \mathrm{l} / \mathrm{min} / \mathrm{m}^{2}$ ) sin encontrar mejoría en los desenlaces ${ }^{36,37}$.

La reanimación hídrica inicial mediante soluciones cristaloides y el uso de vasopresores tempranos son pilares fundamentales en la fase inicial. Para prevenir la sobrecarga hídrica la reanimación debe ser guiada mediante el uso de variables dinámicas, como variación de presión de pulso y variación de volumen sistólico. La elevación pasiva de piernas es el estándar de oro para determinar la respuesta a volumen y tiene la ventaja adicional de que puede realizarse con el paciente en ventilación espontánea ${ }^{38,39}$.

Las guías actuales recomiendan norepinefrina como el vasopresor de primera línea para mejorar la PAM y mediante esto, la presión de perfusión en los pacientes con shock séptico a pesar de un adecuado manejo de líquidos ${ }^{40}$. Los efectos alfaadrenérgicos generan un aumento de la poscarga y disminución ligera del GC, y se acompañan de un efecto betaadrenérgico a nivel de perfusión tisular y de las resistencias vasculares pulmonares ${ }^{41}$. Debido a sus propiedades de aumento de consumo de oxígeno a nivel miocárdico y la incidencia de arritmias, la epinefrina no se considera de primera elección en miocardiopatía séptica ${ }^{42}$. 
La vasopresina es el segundo vasopresor que puede ser utilizado, ya que este actúa a través de los receptores $\mathrm{V}^{42}$. El estudio VASST evaluó el uso de vasopresina en pacientes con shock séptico. Al igual que en otros estudios que se desarrollaron después, el uso de vasopresina fue una opción en el shock séptico refractario, pero no como vasopresor de primera elección ${ }^{43}$. En el contexto de miocardiopatía séptica el uso de vasopresina se asocia con una disminución en la frecuencia cardiaca, pero sin cambios en el GC o marcadores de hipoperfusión ${ }^{44}$.

Con base en los objetivos que se deben llevar a cabo en el paciente con sepsis, la dobutamina no se recomienda de manera sistemática en el manejo de la miocardiopatía séptica, buscando la normalización de un parámetro aislado como la saturación venosa central de oxígeno ${ }^{45}$.

El soporte inotrópico con dobutamina se sugiere cuando la reanimación con líquidos y vasopresores no es suficiente para reestablecer una adecuada perfusión sistémica ${ }^{40,46}$. El uso de dobutamina mejora el GC y el IC pero sin evidencia de mejoría en los desenlaces finales de los pacientes. La dosis óptima de dobutamina en miocardiopatía séptica no se ha establecido adecuadamente, por lo que se pueden llegar a utilizar dosis tan altas como $20 \mu \mathrm{g} / \mathrm{kg} / \mathrm{min}^{40}$. Sin embargo la combinación de dobutamina y norepinefrina no mostró diferencia en la mortalidad a 28 días en comparación con epinefrina ${ }^{47}$. De la misma manera, el levosimendán, medicamento sensibilizador de calcio, con propiedades inotrópicas y lusotrópicas no ha mostrado una disminución en mortalidad o disfunción orgánica en los pacientes con shock séptico y miocardiopatía ${ }^{48}$.

Comparado con la dobutamina, el levosimendán disminuye los niveles de biomarcadores de lesión miocárdica, incluyendo troponina I y BNP, incrementa la FEVI y la función contráctil del VI, así como mejoría de las variables hemodinámicas sistémicas ${ }^{49}$.

Según un metaanálisis llevado a cabo por Chang, et al. ${ }^{50}$, aunque el levosimendán puede mejorar los parámetros clínicos como IC y perfusión tisular comparado con dobutamina o el tratamiento estándar, aún no genera un beneficio estadístico claro en mortalidad en el contexto de sepsis. Se recomiendan más estudios para generar un mejor nivel de evidencia, sobre todo cuando se presenta una disfunción cardiaca de manera franca.

El bloqueo betaadrenérgico se ha introducido como un tratamiento potencial para la cardiomiopatía inducida por catecolaminas. La disminución de la frecuencia cardiaca puede llevar a un aumento del tiempo de llenado diastólico, mejoría en la perfusión coronaria, incremento de la contractilidad y disminución del consumo de oxígeno en sepsis ${ }^{51,52}$.

El aumento del consumo miocárdico de oxígeno causado por la taquicardia es el sustento teórico para controlar la frecuencia cardiaca en pacientes con miocardiopatía séptica. Al respecto un estudio demostró un 31\% de reducción en la mortalidad a los 28 días cuando el rango de frecuencia cardiaca está entre 80 y 94 latidos por minuto, titulado con esmolol ${ }^{53}$. El esmolol incrementaba el volumen latido, manteniendo la PAM, y permitía reducir las dosis de norepinefrina sin efectos deletéreos en otros órganos ${ }^{51}$. Los betabloqueadores pueden modular la respuesta inflamatoria miocárdica mediante la activación de los receptores $\mathrm{B} 2$ y el bloqueo de los receptores $\mathrm{B} 1^{54}$.

\section{Conclusión}

La presencia de falla hemodinámica en el paciente séptico tiene diversa etiología más allá de la pérdida de las resistencias vasculares sistémicas. El desencadenamiento de una respuesta inflamatoria descontrolada lleva a diminución de la función de bomba. Estos cambios habitualmente son discretos en estadios iniciales, por lo cual se requiere una adecuada sospecha diagnóstica y el uso de estudios de imagen como ecocardiografía a la cabecera del paciente. Con el paso del tiempo y el mejor entendimiento de los cambios fisiopatológicos se dispondrá de evidencia más consolidada para el paciente con miocardiopatía séptica.

\section{Financiamiento}

La presente investigación no ha recibido ninguna beca específica de agencias de los sectores público, comercial o sin ánimo de lucro.

\section{Conflictos de interés}

Los autores declaran no tener conflicto de intereses.

\section{Bibliografía}

1. Antonucci E. Myocardial depression in sepsis: From pathogenesis to clinical manifestations and treatment. J Crit Care. 2014;29(4):500-11.

2. Parker M. Profound but reversible myocardial depression in patients with septic shock. Ann Intern Med. 1984;100:483-90.

3. Romero F. Sepsis-induced cardiomyopathy. Current Cardiology Reviews. 2011;7:163-83.

4. Beesley S, Weber G, Sarge T, Nikravan S, Grissom CK, Lanspa MJ, et al. Septic cardiomyopathy. Crit Care Med. 2018;46(4):625-34.

5. Lang RM, Badano LP, Mor-Avi V, Afilalo J, Armstrong A, Ernande L, et al. Recommendations for cardiac chamber quantification by echocardiography in adults: An update from the American Society of Echocardiography and the European Association of Cardiovascular Imaging. J Am Soc Echocardiogr. 2015;28(1):1-39.e14. 
6. Huang SJ, Nalos M, McLean AS. Is early ventricular dysfunction or dilatation associated with lower mortality rate in adult severe sepsis and septic shock? A meta-analysis. Crit Care. 2013;17(3):R96.

7. Sevilla Berrios RA, O'Horo JC, Velagapudi V, Pulido JN. Correlation of left ventricular systolic dysfunction determined by low ejection fraction and 30-day mortality in patients with severe sepsis and septic shock: A systematic review and meta-analysis. J Crit Care. 2014;29(4):495-9.

8. Parker MM, McCarthy KE, Ognibene FP, Parrillo JE. Right ventricular dysfunction and dilatation, similar to left ventricular changes, characterize the cardiac depression of septic shock in humans. Chest. 1990;97(1):126-31.

9. Nagueh SF, Smiseth OA, Appleton CP, Byrd BF $3^{\text {rd }}$, Dokainish H, Edvardsen $\mathrm{T}$, et al. Recommendations for the Evaluation of Left Ventricular Diastolic Function by Echocardiography: An Update from the American Society of Echocardiography and the European Association of Cardiovascular Imaging. J Am Soc Echocardiogr. 2016;29(4):277-314

10. Vieillard A. Actual incidence of global left ventricular hypokinesia in adult septic shock. Crit Care Med. 2008;36(6).

11. Pulido J. Clinical spectrum, frequency, and significance of myocardial dysfunction in severe sepsis and septic shock. Mayo Clin Proc. 2012;87(7):620-8.

12. Landesberg G. Diastolic dysfunction and mortality in severe sepsis and septic shock. Eur Heart J. 2012;33:895-903.

13. Geer L. Variability in echocardiographic measurements of left ventricular function in septic shock patients. Cardiovasc Ultrasound. 2015;13:19.

14. Walley KR. Sepsis-induced myocardial dysfunction. Curr Opin Crit Care. 2018;24(4):292-9.

15. Xiuxiu LV, Huadong W. Pathophysiology of sepsis-induced myocardial dysfunction. Mil Med Res. 2016:3:30.

16. Aneman A, Vieillard-Baron A. Cardiac dysfunction in sepsis. Intensive Care Med. 2016;42(12):2073-6.

17. Antonucci E, Fiaccadori E, Donadello K, Taccone FS, Franchi F, Scolletta S. Myocardial depression in sepsis: From pathogenesis to clinica manifestations and treatment. J Crit Care. 2014;29:500-11.

18. Russell JA, Rush B, Boyd J. Pathophysiology of septic shock. Crit Care Clin. 2018;34:43-61.

19. Riera J, Romay E, Ferrer R. Management of myocardial dysfunction in septic shock. Potential role of extracorporeal membrane oxygenation. Medicina Intensiva. 2018;42(5):301-5

20. Suzuki T, et al. Sepsis-induced cardiac dysfunction and $\beta$-adrenergic blockade therapy for sepsis. J Intensive Care. 2017;5:22.

21. Potz BA, Sellke FW, Abid MR. Endothelial ROS and impaired myocardia oxygen consumption in sepsis-induced cardiac dysfunction. J Intensive Crit Care. 2016;(2):11.

22. Fenton KE, Parker MM. Cardiac function and dysfunction in sepsis. Clin Chest Med. 2016;37(2):289-98

23. Evans DC, Doraiswamy VA, Prosciak MP, et al. Complications associated with pulmonary artery catheters: a comprehensive clinical review. Scand J Surg. 2009;98(4):199-208.

24. Brovman EY, Gabriel RA, Dutton RP, Urman RD. Pulmonary artery catheter use during cardiac surgery in the United States, 2010 to 2014 J Cardiothorac Vasc Anesth. 2016;30(3):579-84.

25. Chatterjee K. The Swan-Ganz catheters: past, present, and future A viewpoint. Circulation. 2009:119(1):147-52.

26. Jozwiak M, Persichini R, Monnet X, Teboul JL. Management of myocardial dysfunction in severe sepsis. Semin Respir Crit Care Med. 2011;32(2):206-14

27. Ganter MT, Alhashemi JA, Al-Shabasy AM, et al. Continuous cardiac output measurement by un-calibrated pulse wave analysis and pulmonary artery catheter in patients with septic shock. J Clin Monit Comput. 2016;30(1):13-22.

28. Krishnagopalan S, Kumar A, Parrillo JE, Kumar A. Myocardial dysfunction in the patient with sepsis. Curr Opin Crit Care. 2002;8(5):376-88

29. Charpentier J, Luyt CE, Fulla Y, et al. Brain natriuretic peptide: A marker of myocardial dysfunction and prognosis during severe sepsis. Crit Care Med. 2004;32(3):660-5.

30. Masson S, Caironi P, Fanizza C, et al. Sequential N-terminal pro-b-type natriuretic peptide and high-sensitivity cardiac troponin measurements during albumin replacement in patients with severe sepsis or septic shock. Crit Care Med. 2016;44(4):707-16.

31. Guarracino F, Baldassarri R, Pinsky MR. Ventriculo-arterial decoupling in acutely altered hemodynamic states. Crit Care (London, England). 2013;17(2):213.
32. Helle-Valle T, Crosby J, Edvardsen T, et al. New noninvasive method for assessment of left ventricular rotation: speckle tracking echocardiography. Circulation. 2005;112(20):3149-56.

33. Ng PY, Sin WC, Ng AK, Chan WM. Speckle tracking echocardiography in patients with septic shock: a case control study (SPECKSS). Crit Care (London, England). 2016;20(1):145

34. Werdan K, Oelke A, Hettwer S, et al. Septic cardiomyopathy: hemodynamic quantification, occurrence, and prognostic implications. Clin Res Cardiol. 2011;100(8):661-8.

35. Wilhelm J, Hettwer S, Schuermann M, et al. Severity of cardiac impairment in the early stage of community-acquired sepsis determines worse prognosis. Clin Res Cardiol. 2013;102(10):735-44.

36. Hayes MA, Timmins AC, Yau EH, et al. Elevation of systemic oxygen delivery in the treatment of critically ill patients. $\mathrm{N}$ Engl $\mathrm{J}$ Med. 1994;330:1717-22.

37. Gattinoni L, Brazzi L, Pelosi P, et al. A trial of goal-oriented hemodynamic therapy in critically ill patients. SvO2 Collaborative Group. N Engl J Med. 1995;333:1025-32.

38. Monnet X, Marik P, Teboul JL. Passive leg raising for predicting fluid responsiveness: a systematic review and meta-analysis. Intensive Care Med. 2016;42(12):1935-47.

39. Preau S, Saulnier F, Dewavrin F, Durocher A, Chagnon JL. Passive leg raising is predictive of fluid responsiveness in spontaneously breathing patients with severe sepsis or acute pancreatitis. Crit Care Med. 2010;38(3):819-25.

40. Rhodes A, Evans LE, Alhazzani W, et al. Surviving sepsis campaign: International guidelines for management of sepsis and septic shock 2016. Crit Care Med. 2017;45(3):486-552.

41. De Backer D, Scolletta S. Clinical management of the cardiovascular failure in sepsis. Curr Vasc Pharmacol. 2013;11(2):222-42.

42. Antonucci E, Fiaccadori E, Donadello K, Taccone FS, Franchi F, Scolletta S. Myocardial depression in sepsis: from pathogenesis to clinical manifestations and treatment. J Critical Care. 2014;29(4):500-11.

43. Russell JA, Walley KR, Singer J, et al. Vasopressin versus norepinephrine infusion in patients with septic shock. N Engl J Med. 2008;358(9):877-87.

44. Gordon AC, Wang N, Walley KR, Ashby D, Russell JA. The cardiopulmonary effects of vasopressin compared with norepinephrine in septic shock. Chest. 2012;142(3):593-605.

45. Nagueh SF, Appleton CP, Gillebert TC, et al. Recommendations for the evaluation of left ventricular diastolic function by echocardiography. J Am Soc Echocardiogr. 2009;22:107-33.

46. Ponikowski P, Voors AA, Anker SD, et al. 2016 ESC Guidelines for the diagnosis and treatment of acute and chronic heart failureThe Task Force for the diagnosis and treatment of acute and chronic heart failure of the European Society of Cardiology (ESC). Developed with the special contribution of the Heart Failure Association (HFA) of the ESC. Eur Heart J. 2016;37(27):2129-200.

47. Annane D, Vignon $P$, Renault $A$, et al. Norepinephrine plus dobutamine versus epinephrine alone for management of septic shock: a randomised trial. Lancet (London, England). 2007;370(9588):676-84.

48. Gordon AC, Perkins GD, Singer M, et al. Levosimendan for the prevention of acute organ dysfunction in sepsis. N Engl J Med. 2016; 375(17):1638-48.

49. Meng, et al. Levosimendad versus dobutamine in myocardial injury patients with septic shock: a randomized controlled trial. Med Sci Monit. 2016;22:1486-946.

50. Chang W, et al. Effect of levosimendan on mortality in severe sepsis and septic shock: a meta-analisis of randomized trial. BMJ Open. 2018;8e019338.

51. Morelli A, Ertmer C, Westphal M, et al. Effect of heart rate control with esmolol on hemodynamic and clinical outcomes in patients with septic shock: a randomized clinical trial. JAMA. 2013;310:1683-91.

52. Lanspa M, Shahul S, Hersh A, et al. Associations among left ventricular systolic function, tachycardia, and cardiac preload in septic patients. Ann Intensive Care. 2017;7:17.

53. Morelli A, Ertmer $\mathrm{C}$, Westphal $\mathrm{M}$, et al. Effect of heart rate control with esmolol on hemodynamic and clinical outcomes in patients with septic shock: a randomized clinical trial. JAMA. 2013;310(16):1683-91.

54. de Montmollin E, Aboab J, Mansart A, Annane D. Bench-to-bedside review: Beta-adrenergic modulation in sepsis. Crit Care. 2009;13(5):230. 